\title{
Civilisations
}

Revue internationale d'anthropologie et de sciences

humaines

66 | 2017

L'alcool rituel et les ethnographes

\section{Transformation de la consommation d'alcool chez les Nanaïs du bassin de l'Amour}

\section{Anne Dalles}

\section{OpenEdition}

\section{Journals}

Édition électronique

URL : http://journals.openedition.org/civilisations/4344

DOI : 10.4000/civilisations.4344

ISSN : 2032-0442

\section{Éditeur}

Institut de sociologie de l'Université Libre de Bruxelles

\section{Édition imprimée}

Date de publication : 31 août 2017

Pagination : 77-90

ISBN : 978-2-9602017-1-0

ISSN : 0009-8140

Référence électronique

Anne Dalles, "Transformation de la consommation d'alcool chez les Nanaïs du bassin de l'Amour », Civilisations [En ligne], 66 | 2017, mis en ligne le 31 août 2020, consulté le 25 février 2021. URL : http:// journals.openedition.org/civilisations/4344; DOI : https://doi.org/10.4000/civilisations.4344 


\title{
Transformation de la consommation d'alcool chez les Nanaïs du bassin de l'Amour
}

\author{
Anne DALLES
}

Résumé : L'utilisation ancienne de la vodka dans les pratiques chamaniques, chez les Nanaïs de l'Extrême-Orient de la Sibérie, variait en fonction du rite : tantôt destinée aux esprits, souvent par le biais du chamane, lors des rites funéraires, de pêche ou encore de commémoration des morts, tantôt objet d'échange, lors des rites de mariages, la vodka soulignait le contrôle du spécialiste sur lui-même, apportait l'apaisement des esprits et affirmait le statut social. Dans le contexte moderne, dans cette population très russisée, dont les grands rites publics ne se font plus, la consommation d'alcool a changé de symbolisme, rentrée désormais dans la sphère privée, et fait même l'objet d'un combat dans l'implantation de nouveaux mouvements religieux. L'exposition de l'usage des libations dans les rites nanaïs anciens, dans un premier temps, suivi, dans un deuxième temps, de la comparaison avec sa perception aujourd'hui, permettent de montrer en quoi la consommation d'alcool depuis la disparition des chamanes est liée à ce qui a survécu des pratiques chamaniques, tout en étant aussi liée à la définition de l'alcool par le groupe culturel dominant russe.

Mots-clés : Nanaïs, alcool, rites chamaniques, alcoolisme.

\begin{abstract}
Among the Nanai of the Siberian Far East, the use of vodka used to change depending on the rites: sometimes intended for the spirits, mostly with the help of the shaman, during the funerary rites, the commemorations for the dead, or the fishing rites, sometimes an object of exchange, for marriage rites, vodka emphasized the religious specialist's control of himself, brought peace with the spirits and confirmed social status. In the modern context, as the Nanai have become russianized and have stopped performing their public rituals, the symbolism of alcohol consumption has shifted, now entering private life, and even constitutes a target in the implantation of new religious movements. The analysis of the usage of libations followed by the comparison of its perception today allows us to show how alcohol consumption has been linked to what has survived in the shamanistic practices since the disappearance of shamans, while being also related to the definition of alcohol by the culturally dominating Russian group.
\end{abstract}

Keywords: Nanai, alcohol, shamanistic rituals, alcoholism. 


\section{Introduction}

Les Nanaïs habitent dans la région de l'Amour, à l'Extrême-Orient de la Sibérie, en Russie. Leur population totale s'élève à environ 12.000 individus, principalement répartis dans la région de Khabarovsk («Vserossijskaja perepis' naselenija 2010 goda » 2010). Ils représentent moins de $1 \%$ de la population de la région et vivent principalement dans des villages ${ }^{1}$. Jusqu'à la collectivisation soviétique, les Nanaïs vivaient de pêche annuelle et de chasse hivernale ; depuis, la pêche constitue encore une part importante des ressources. Suite à la politique antireligieuse mise en place pendant la période soviétique, les grands rites publics qui faisaient la particularité du chamanisme nanaï ne se font plus depuis les années 1970, et il n'y a plus de chamane dit « traditionnel». De nombreuses pratiques, liées à la gestion des esprits chamaniques et des âmes, ont été adaptées pour pallier l'absence de chamane.

L'utilisation de libations d'alcool en contexte rituel permettait de négocier la chance avec les esprits, de mettre en avant le contrôle du spécialiste religieux, le chamane, sur les esprits et le statut social des différentes parties impliquées dans le rite dans certains cas. Ces contextes permissifs de consommation (parfois excessive) d'alcool se retrouvent désormais dans les pratiques chamaniques qui persistent malgré l'absence de chamane. Parallèlement, la consommation d'alcool est désormais aussi perçue par le groupe dominant (numériquement et culturellement), les Russes, en termes d'alcoolisme et cause de suicide.

Interprétés localement ${ }^{2}$ comme les conséquences de l'absence de chamane et de l'errance des âmes, l'alcoolisme, le suicide et la dépression, symptomatiquement liés dans la médecine occidentale (Scheper-Hughes et Locke 1987), constituent par ailleurs, et ce depuis au moins un demi-siècle, un problème d'envergure chez les populations autochtones de Russie. D'après nos analyses, il apparaît que la place de l'alcool, dans une définition culturelle tantôt russe, liant le phénomène au suicide et à la dépression, tantôt nanaïe, associée au poids des âmes et à l'errance des esprits, est reflétée dans une utilisation double et paradoxale de la boisson, conduisant à l'identification de différents contextes de consommation. Comment appréhender ce phénomène, qui pose de vraies questions de santé publique, et semble s'inscrire dans une multi-layered definition (Haas 2014 ; Obadia 2004a)?

L'analyse de l'utilisation rituelle de l'alcool dans le cadre du cycle des funérailles et des anciens rites de mariage nanaïs fait d'abord apparaître l'alcool comme outil de

1 Les Nanaïs font partie des Peuples Minoritaires (Indigènes) du Nord, de la Sibérie et de l'Extrême-Orient de la Fédération de Russie, catégorie administrative qui regroupe environ 40 minorités ethniques de Russie de moins de 50.000 individus. La grande majorité de la population dans la région de l'Amour est russe.

2 Mon travail de terrain s'est déroulé durant 7 mois entre 2011 et 2015 dans la région de l'Amour en Russie et en Chine dans le cadre d'une thèse portant sur les pratiques religieuses contemporaines en lien avec la gestion des âmes, les transformations rituelles et la conversion chez les Nanaïs. Un premier terrain a été financé par le Pentecostal and Charismatic Research Initiative ainsi qu'un second, en Chine, par le programme Dynamiques Asiatiques (Pres. Hesam). Les données ont été récoltées à partir d'enquêtes qualitatives, principalement par observation directe (souvent participante), donnant également lieu à des entretiens non directifs et semi-directifs auprès d'environ cinquante personnes (en anglais et en russe). Mes enquêtes ont pris place dans les trois grandes villes et quatre villages du Kraj de Khabarovsk en Russie, et dans cinq villes et villages de la région du Heilongjiang en Chine. Dans le cadre de cet article, les discours sur l'alcool faisant l'objet d'une certaine gêne, je respecte l'anonymat de mes informateurs. 
négociation de chance, tantôt auprès des esprits qu'il faut amadouer, tantôt auprès des hommes dans les interactions sociales. Ensuite, la consommation d'alcool dans le cadre plus large de la question de l'alcoolisme en Russie est aujourd'hui associée à des notions de santé publique et de pathologie. La " manière de boire », plutôt que la quantité ou la qualité de l'alcool, caractérise alors l'ambivalence de cette consommation, entre offrande aux esprits et alcoolisme.

\section{Amadouer et contrôler les esprits}

Jusque dans les années 1970, le cycle des funérailles nanaïes, composé des funérailles en elles-mêmes puis de trois rites visant à faire parvenir l'âme du défunt au monde des morts, pouvait durer plusieurs années (Lopatin 1960 ; Shimkevich 1896 ; Sternberg 1933). Coûteux, le traitement rituel du mort nécessitait nombre de libations, constituées de tabac, nourriture rituelle et boissons, afin de faire patienter l'âme avant qu'elle ne quitte le monde des vivants. La durée du cycle (jusqu'à cinq ans pour les familles les plus modestes) et le choix des boissons dépendaient de la richesse des proches du défunt.

Lors de ces rites, plusieurs moments étaient consacrés à la consommation d'alcool partagée entre le défunt, les esprits, le chamane et les personnes présentes. Pendant les funérailles, les proches du défunt pouvaient boire de la vodka avec lui en versant un peu du liquide sur sa poitrine (Gaer 1991 : 91-111 ; Smoljak 1980a ; Smoljak 1968). Après avoir été habillé de somptueux vêtements brodés, le défunt recevait les offrandes de nourriture et de boisson : " tu es mort, nous ne te reverrons plus, s'il te plait, découvre ces plats » (Sternberg $1933: 478$ ).

Une fois le défunt enterré, l'alcool était versé dans un petit trou proche du feu dans lequel les offrandes pour le mort étaient jetées (Smoljak 1968 ; Gaer 1991). Pendant les rites commémoratifs, les proches partageaient les libations avec le défunt par l'intermédiaire d'une figure anthropomorphe en bois, dans laquelle le chamane plaçait l'âme du mort ; la vodka était alors versée dans un petit trou devant la figurine ou directement sur ses lèvres (Shimkevich 1896 : 22-33 ; Lopatin 1960 : 138-147 ; Gaer 1991 : 91-111). En transmettant les offrandes lors du rite final, le chamane exhortait le défunt à quitter les vivants (Sternberg 1933 : 483-490).

Le chamane utilisait aussi de la vodka comme libation pour ses esprits auxiliaires censés l'aider à accomplir les rites. Les hommes, venus inviter le chamane à accomplir les rites funéraires, lui offraient de la vodka, qu'il consommait et aspergeait sur son costume à plusieurs reprises à l'attention de ses esprits auxiliaires. Arrivé sur les lieux du rite, on offrait à nouveau de la vodka au chamane, assis à la place d'honneur (Shimkevich 1896 : 22-33). En aspergeant ses accessoires de vodka, le chamane appelait ses esprits : "Venez tous ici ! Faisons une grande fête ; buvons de la vodka, et mangeons, et soyons heureux ! L'hôte a préparé beaucoup de vodka et de tabac pour nous. Volez vers moi ! Je vous appelle!»(Lopatin 1960 : 160-173). Le chamane devait prouver qu'il contrôlait sa relation avec les esprits constamment, afin d'assurer la réussite du rite : devant trouver l'âme du mort pour la placer dans son support et la guérir par la suite, un chamane inefficace pouvait être éconduit après avoir été battu pour s'être trompé d'âmes à plusieurs reprises (Lopatin 1960 : 138-147). 
Les grands rites publics du cycle des funérailles nanaïs ne sont plus effectués depuis les années 1970 et il n'existe plus de chamanes dits « traditionnels $»^{3}$ depuis les années 1990 (Smoljak 1991 ; Bulgakova 2003 ; Bulgakova 2013). Selon les villages, les funérailles ont été aménagées différemment afin de pallier l'absence de chamanes, tandis que les Nanaïs les plus âgés peuvent garder chez eux une figuration du défunt. Ainsi, les photographies ont pris la place des figures en bois anthropomorphes et l'on effectue quelques libations devant celles qui se trouvent sur les tombes (Smoljak 1968 ; Gaer 1983 ; Bulgakova 2013). Après l'enterrement, les proches peuvent garder un bâtonnet (Gaer 1991 : 104) ou un morceau de ficelle accroché à un bouton qu'ils tremperont dans l'alcool et la nourriture lors des libations à la maison. Ailleurs, les proches retournent sur la tombe une fois par an pour faire des offrandes aux défunts, ce qui était interdit auparavant.

Nourrir les esprits avec de la vodka comptait parmi les obligations du chamane et un oubli pouvait s'avérer dangereux, voire fatal. Le chamane Nikolaï Petrovitch a ainsi raconté à l'ethnographe Tatiana Bulgakova dans les années 1990 :

« J'avais bu et je revins à la maison avec une bouteille. Je m'assis à la table, ouvris la bouteille. N'étais-je pas jeune à cette époque ? Je versai (de la vodka) pour moi, mais ne mis rien dans le coin (pour le seven ${ }^{4}$ ). Je bus et allai me coucher. Comme ça me frappa la nuit ! Je tremblais ! Ça me prit si soudainement ! Mais que pouvais-je faire? Il n'y avait pas de vodka du tout » (Bulgakova 2013 : 140).

Une histoire similaire fut racontée par sa femme, la chamane Mingo :

« J'ai oublié de mettre (de la vodka pour mes seven) et j'ai failli en mourir. J'ai mis des pâtes dans de l'eau bouillante, mais je n'ai même pas eu le temps de mettre le couvercle sur la casserole, quand je suis tombée et j'ai perdu conscience » (Bulgakova 2013 : 140-41).

La vodka avait parfois aussi une origine inconnue, voire surnaturelle. Lors d'une séance rapportée par une chamane dans les années 1990, une femme cherchait des remèdes à sa maladie en dansant sur des charbons ardents ; de la vodka apparût mystérieusement dans les petits verres disposés pour recevoir le remède : «Qui avait versé le remède [vodka] dans ces verres vides ? Quelle nuit ! Quelle longue nuit !» (Bulgakova 2013: 31).

3 Selon la traduction du terme utilisé localement et par opposition aux quelques néo-chamanes nanaïs qui travaillent dans la région. Ces derniers ont une interprétation alliant psychiatrie, psychologie et médecine occidentale de leur pratique, et utilisent un vocabulaire commun à d'autres néo-chamanes dans le monde ( fluides », « canaux », " énergies ») mais de nombreux éléments restent proches des pratiques chamaniques pensées comme «traditionnelles» (héritage chamanique, esprits animaux, âmes des morts) [informations recueillies à Khabarovsk en novembre 2013] (Bulgakova 2013: 220). Pour T. Bulgakova, le néo-chamanisme nanaï est également lié à une forme de revivalisme culturel soulignant une volonté de retour aux pratiques anciennes liée à une mise en avant du caractère national (au sens ethnique) du chamanisme (Bulgakova 2008). Ce dernier point se retrouve également dans le néo-chamanisme en Mongolie, où la pratique chamanique semble étroitement liée à la perception d'une culture mongole authentique ancrée dans le passé (à l'époque de Chinggis Khan), combinant énergies personnelles et pouvoir national (Merli 2004a; Merli 2004b).

4 Esprit chamanique. 


\section{Négocier son statut social}

Négociateur de chance, l'alcool était consommé à profusion lors des rites liés au mariage effectués jusqu'aux années 1930. Le cycle du mariage nanaï, fonctionnant selon un système d'alliance asymétrique (Lévi-Strauss 1967 ; Sternberg 1999), comprenait plusieurs visites du clan du marié lors desquelles s'effectuaient les négociations du prix de la fiancée et du contenu du trousseau et l'emménagement de la mariée (Smoljak 1980b ; Gaer 1991 : 68-89; Samar 2003). Objet d'échange précieux, l'alcool rentrait dans le coût informel payé par le clan du futur mari, comme preuve de richesse et pour consoler le clan qui allait se séparer d'une de ses filles. Il pouvait aussi servir d'unique apport pour les familles les plus modestes.

Le fiancé pouvait apporter jusqu'à 20 litres de vodka à chacune des visites, consommée une fois la demande en mariage acceptée (Smoljak 1980b). La vodka était servie dans de la vaisselle précieuse aux membres des deux clans et aux esprits. Quand le fiancé revenait avec le montant demandé , le couple se présentait aussi en tant que tel pour la première fois : la fiancée tenait la bouteille, tandis que le fiancé servait de la vodka à tous, en s'agenouillant devant les anciens afin d'attirer leur bienveillance (Smoljak 1980b ; Gaer 1991).

Après avoir quitté la maison paternelle, la fiancée servait un dernier verre à ses parents avant de monter dans la barque qui l'emmènerait dans sa nouvelle maison; ses parents offraient alors un peu d'alcool aux esprits en y trempant un doigt et en en secouant à terre, au milieu des lamentations (Gaer 1991 : 77-80). A l'arrivée dans sa nouvelle maison, la jeune femme et son nouveau mari servaient de la vodka et recevaient les souhaits des anciens : « Et nous buvons la vodka du mariage ! Vivez heureux ! Ne vous querellez pas !» (Gaer $1991: 83)$.

Le cycle des mariages traditionnels nanaïs n'existe plus depuis les années 1930 et les mariages par achat ont graduellement été interdits pendant la période soviétique (LéviStrauss 1967 ; Bacon 1980 ; La coutume (4) 1990 : 147 ; Slezkine 1994 : 102 ; Balzer 1999 ; Sternberg 1999 ; Samar 2003 : 100 ; Bulgakova 2013 : 216 ; Berelowitch 1981). Tandis qu'officiellement, la volonté était de combattre les coutumes locales et de faire du mariage une simple procédure civile (Berelowitch 1981 : 357-59 ; La coutume (4) 1990 : 147), certains Nanaïs y voyaient un lien avec la consommation d'alcool. Un Nanaï chamane non-initié confiait ainsi à l'ethnographe T. Bulgakova :

« Non, les mariages n'étaient pas interdits, mais c'était interdit de se saouler. Quand ils attrapaient une personne saoule, ils le condamnaient aux camps de travail. [...] Donc les mariages n'avaient plus lieu. Les gens s'asseyaient un peu à la maison, en buvant un coup et c'est tout ! [...] [Jusqu'aux années 1930], la mariée était la première à partir [vers le village de son mari]. Ils arrivaient et offraient de la vodka à tout le monde. Et on ne pouvait pas refuser ! [...] mais sous le gouvernement soviétique, les gens ont arrêté de faire tout ça. Ils avaient peur!» (Bulgakova $2013: 216)$.

5 Le prix de la mariée dépendait du statut social de la famille de la jeune fille, et pouvait être payé en plusieurs fois. Ce paiement était constitué de fourrures, bijoux, soieries, objets sculptés, etc. (Zolotarev 1939 : 54-62 ; Smoljak 1980b ; Gaer 1991 : 68-90). 
Les Nanaïs célèbrent désormais les mariages à la mairie puis chez l'un des parents des mariés (ou au restaurant en ville), comme le font également les Russes. Les mariés ne doivent plus forcément changer de village et les échanges inter-claniques selon le système asymétrique ne se font plus. L'alcool a toute sa place dans ces nouvelles pratiques et la pratique du " toast » russe permet le maintien de la négociation de chance et de statut social. Certains éléments anciens sont conservés plus ou moins implicitement, comme offrir à la mariée un nécessaire de couture ${ }^{6}$.

\section{L'alcoolisme de nos jours}

Dans le cadre plus large de la question de l'alcoolisme en Russie, la consommation excessive d'alcool et le suicide sont considérés comme des pathologies. Selon cette perception, les populations autochtones de Russie sont considérées comme des groupes particulièrement à risque.

Dès le $17^{\mathrm{e}}$ siècle, lorsque l'Etat prenait le monopole de la production et de la vente d'alcool en Russie, sa consommation était influencée par les politiques en vigueur (White 1996 ; Takala 2002 ; Schrad 2014 ; McKee 1999 ; Kossov 2014). L'alcool constituait alors une part importante des revenus de l'Etat, mais il faisait aussi l'objet de campagnes anti-alcool, particulièrement à partir de la période soviétique. Objet d'échange avec les populations autochtones (Atkinson 2001 ; Smoljak 1975 ; Smoljak 2005), l'alcool avait aussi une dimension officieuse, liée à la production domestique de samogon "7, qui, lors de ces campagnes, intégrait une économie parallèle. La perception de la consommation d'alcool comme facteur de problèmes sociaux, de santé ou encore comme cause du manque de productivité (à l'origine des échecs des politiques économiques) était à la base des campagnes anti-alcool depuis les années 1980 (McKee 1999 ; Walberg et al. 1998 ; Treml 1997 ; Kossov 2015).

De nombreuses études tentent difficilement d'estimer la consommation d'alcool en Russie et parmi les populations autochtones. Ces études sont confrontées aux limites des outils utilisés pour ces estimations : statistiques officielles compliquées, faussées, inexistantes, taux non-enregistrés, sous/sur évaluation de la consommation, etc. Les taux d'alcoolisme en Russie sont élevés : $20 \%$ de la population aurait des épisodes de « lourde $»^{8}$ consommation d'alcool tous les mois, dont $30 \%$ de la population masculine adulte (World Health Organization 2014 ; World Health Organization 2011b). La qualité de la vie en Russie est directement concernée. Une forte corrélation existe entre la consommation d'alcool (estimée) et les taux de mortalité liés à l'alcool (accidents cardio-vasculaires, accidents, crimes et suicides) (Bobak et al. 1999 ; Nemtsov 2009 ; Walberg et al. 1998). On estime à 16,8\% les nombre de décès liés à l'alcool en l'Extrême-Orient russe (Nemtsov 2009). L'analyse des «façons de boire » soulignent des pratiques culturelles dominantes, considérées comme dangereuses : le zapoi, ou l'hyper-alcoolisation rapide, la consommation d'alcools de substitution, ou encore la distillation d'alcools de mauvaise qualité auraient un impact direct sur les taux de

6 Informations recueillies à Najhin, Sikachi Alyan et Khabarovsk.

7 Nom communément donné à l'alcool produit domestiquement. Généralement fabriqué selon des recettes locales (voire familiales), il contient du sucre et des fruits (Treml 1997 ; McKee 1999; Nemtsov 2011 ; Solodun et al. 2011). Chez les Nanaïs, le samogon' est généralement à base de baies sauvages.

Consommation d'au moins 60 grammes d'alcool pur en une occasion (WHO, 2014). 
mortalité (Treml 1997 ; Nemtsov 2011 ; Bobak et al. 1999).

Les taux d'alcoolisme et de suicides chez les " populations autochtones » de Russie sont très supérieurs à la moyenne russe. La consommation d'alcool aurait un effet direct sur la composition de la population chez ces groupes : les hommes en âge de travailler (18-59 ans) représentent le groupe le plus à risque (Pivneva 2005 ; Bogojavlenskij 2008). Selon une étude médicale sur les malades atteints d'alcoolisme et de psychose alcoolique dans le kraj de Khabarovsk, le nombre de patients issus des minorités représente le double de celui de la population russe $(25,41 / 100000$ contre 13,87 / 100000) (Mostovoj et Polozhij 1998).

De nombreuses études mettent en lien pauvreté ou conditions de vie difficiles comme facteur aggravant de l'alcoolisme (Ryan 1995). Localement, cette interprétation est aussi prédominante : l'isolement et le chômage sont les premières raisons mises en avant (Kosareva et Mostovoj 1998 ; Kosareva et Panjushkina 1998) ${ }^{9}$. Cependant, le rôle économique et social de la vodka en Russie a largement contribué à une forte consommation d'alcool, et celle-ci ne s'applique pas seulement aux classes les plus démunies (Kossov, 2014 ; McKee, 1999 ; Nemtsov, 2000 ; Nemtsov, 2009 ; Treml, 1997 ; White, 1996). Source importante de revenus pour l'Etat à différentes périodes, tantôt stimulée tantôt prohibée, la consommation d'alcool a une place culturelle en Russie qu'il faut détacher de la simple explication d'un alcoolisme pathologique lié aux conditions sociales. L'analyse des rapports médicaux et scientifiques publiés par plusieurs structures de la région de Khabarovsk (Pozolotin 2014 ; Kolotilin et Kudrjavcev 1998 ; Kolotilin et al. 1998) montre que les « façons de boire » sont en réalité différentes entre les populations autochtones de la région et les Russes. Ailleurs en Russie, l'analyse de la consommation d'alcool, des taux de suicides et de mortalités pour causes externes a été réalisée via des éléments culturels précis (saisonnalité, activité socio-économique, identification de situations difficiles) (Istomin 2012 ; Sumarokov et al. 2014 ; Pika, Davis, et Krupnik 1993 ; Pivneva 2005 ; Ziker 2002). La place de l'alcool et du suicide chez les populations autochtones relèverait de critères culturels propres, différents de ceux associés à ces mêmes phénomènes chez les Russes.

\section{La place ambivalente de l'alcool}

L'étude de la place ambivalente de l'alcool auprès de plusieurs groupes dans le monde montre que sa consommation peut être aussi bien socialisante que désocialisante dans un même contexte (Haas 2014 ; Obadia 2004a ; Obadia 2004b). La définition de l'alcoolisation en termes tantôt négatifs tantôt positifs varie en fonction de la « manière de boire » plutôt que de la quantité ou de la qualité de boisson ingérée, comme l'a montré Obadia au Népal (Obadia 2004b). Les contextes rituels de consommation d'alcool permissifs contemporains correspondent aux contextes anciens de fêtes et de rites, en lien avec la négociation avec les esprits et le statut social. Par opposition, les situations de consommation associée à l'alcoolisme sont liées à une consommation de «type

9 Ces situations perçues comme « difficiles » correspondent à certaines situations professionnelles (la pêche ou le chômage), sociales (pauvreté) ou relationnelles (célibat). La notion de « difficulté » est comprise ici comme subjective, sachant que d'autres situations, perçues comme " non difficiles » ou plus avantageuses, comme les métiers de l'enseignement, ne correspondent pas pour autant à une amélioration nette des conditions de vie. 
russe ». Ainsi, la consommation domestique de vodka ou de samogon' sur le mode du zapoi ou hyper-alcoolisation rapide est à contraster avec les manières de boire rituelles, soulignées précédemment, plus graduelles et liées à la consommation perçue des esprits. Tandis que la quantité d'alcool et l'ivresse peuvent être assez proches dans les deux cas, l'hyper-alcoolisation rapide de "type russe » est considérée comme inacceptable, et s'avère pourtant élevée dans les villages nanaïs. De plus, d'autres types d'alcool (vin ou bière) sont consommés dans des foyers qui condamnent l'alcoolisme. L'ambivalence entre l'alcool de socialisation et la surconsommation condamnée publiquement (Kossov 2014 ; Kossov 2015) crée alors une situation anxiogène, en lien avec des questions de positions professionnelles (les responsabilités politiques ou sociales), de socialisation commerciale, d'hospitalité et de pathologie (Haas 2014 ; Lacaze 2004 ; Obadia 2004a ; Obadia 2004b).

Aujourd'hui, nombre de mes informateurs attribuent l'alcoolisme aux mauvaises conditions de vie. Parallèlement, l'alcoolisme est aussi mis en relation avec l'errance des âmes des morts qui n'ont pas pu atteindre le monde des morts et des esprits chamaniques sans maître depuis la disparition des chamanes. Ainsi, la réponse à la situation de « détresse », soit la place des esprits néfastes conséquente à l'absence de chamane, semble donc être devenue l'alcoolisme et le suicide. Selon plusieurs informateurs de T. Bulgakova, ce sont les esprits et les combats entre chamanes qui auraient causé l'alcoolisme d'une femme chamane décédée dans les années 1990 (Bulgakova 2003 : 148). Une femme nanaïe de Najhin confiait aussi : « Il faudrait un chamane vraiment très fort pour vaincre l'alcoolisme ${ }^{10}$. Cette consommation fait miroir à celle considérée comme « acceptable », en contexte rituel ou festif et met en valeur l'identification de différents contextes selon des définitions tantôt nanaïes, tantôt russes.

L'alcoolisme selon une définition russe (hyper-alcoolisation, liée à certains taux de mortalités, impact sanitaire et social, etc.), en lien direct avec la dépression et le suicide, est considéré comme une pathologie, et rentre dans ce que certains chercheurs appellent les « idiomes de détresse » (Nichter 1981 ; Massé 1999 ; Massé 2007 ; Keys et al. 2012 ; Hinton et Lewis-Fernandez 2010). L'alcoolisme comme expression culturelle de la « détresse » semble se retrouver chez les Nanaïs. Le passage de la consommation rituelle collective à la consommation individuelle, marqué par la variation des « manières de boire », notamment le zapoi, peut permettre alors d'identifier la différence entre consommation d'alcool et alcoolisme, entre alcoolisation festive et alcoolisationmaladie.

\section{Eglise, conversion et alcoolisme}

Certaines représentations collectives des Nanaïs mentionnent le concept d'alcoolisme endémique comme prégnant. Cette idée est véhiculée par les Russes, les médias et les Nanaïs eux-mêmes, sans toutefois donner de mesure de la consommation. Des liens automatiques entre Nanaïs et alcoolisme font alors l'objet de discours sur les Nanaïs, notamment sur les pêcheurs, les personnes sans emploi et les femmes. 
Lors d'une conversation au sujet de l'alcoolisme chez les Nanaïs en 2015, un prêtre orthodoxe russe, qui fréquente les Nanaïs dans les villages depuis son enfance, et effectue des missions dans les villages nanaïs depuis plusieurs années, a immédiatement souligné l'alcoolisme féminin comme un problème symptomatique des « populations autochtones » de la région. Ce prêtre parlait également de la violence conjugale exercée par les femmes alcoolisées chez les Nanaïs ${ }^{11}$.

Une ancienne institutrice russe, rencontrée à Khabarovsk, qui avait travaillé dans le district nanaï dans les années 1980, racontait en 2015 une anecdote tout aussi révélatrice de la vision des Nanaïs :

«Elle travaillait à l'école d'un village russe, situé à quelques centaines de mètres d'un village nanaï, où les enfants venaient étudier. A la recherche d'un enfant nanaï absent de l'école, elle arriva dans le village devant une maison à la porte entrebâillée ; quand elle rentra dans la pièce, elle trouva une femme ivre endormie sur le lit, un petit enfant de quelques mois à côté d'elle jouant avec une bouteille de vodka vide. Elle prit l'enfant pour l'amener à la maison voisine où elle trouva des gens réunis qui lui dirent qu'ils garderaient le petit le temps que la mère dessaoule $»^{12}$.

Les représentations sociales dans lesquelles s'inscrivent ces deux exemples attribuent aux « populations autochtones » certaines spécificités comportementales liées à la consommation d'alcool. L'ivresse des femmes, la santé mentale détériorée des enfants et les conséquences néfastes sur l'environnement familial deviennent des particularités propres à ces populations (Pivneva 2005).

Plus encore, le problème de l'alcoolisme donne lieu à des actions de la part de certains groupes, notamment par des Eglises évangéliques, présentes dans la région depuis les années 1990. Dans le village nanaï de Najhin, il existe un internat dans lequel les orphelins, les enfants séparés des parents ou ceux qui viennent à l'école du village (en très petit nombre) sont logés. Tous les informateurs interrogés s'accordent à dire que la majorité des enfants sont dans cet établissement à cause de l'alcoolisme des parents. Le prêtre orthodoxe russe, qui travaille beaucoup avec ces enfants, souligne également le lien entre abandon des enfants et alcoolisme des parents. Dans une église évangélique charismatique de Khabarovsk, une situation confuse est survenue en lien avec l'adoption d'enfants des villages de la région : plusieurs couples américains seraient venus pour adopter un enfant. Certains seraient passés par leurs contacts dans les églises évangéliques. Quelques années plus tard, à l'adolescence, ces enfants auraient développé des troubles du comportement que tout le monde a attribué à l'alcoolisme de la mère (pendant la grossesse ou pendant la petite enfance) ${ }^{13}$. Les couples adoptants auraient alors voulu rendre les enfants aux orphelinats. En conséquence, les couples

11 Informations recueillies lors d'un entretien informel à Khabarovsk en avril 2015.

12 Informations recueillies lors d'un entretien informel à Khabarovsk en avril 2015.

13 Il est reconnu médicalement que consommer de l'alcool pendant la grossesse est dangereux et peut conduire à un retard mental ou physique de l'enfant, des troubles du comportement, ou encore à des cas de fœtopathie alcoolique (« Les enfants qui en sont atteints naissent avec des déficiences physiques et mentales caractéristiques - petite taille, petite tête et petit cerveau ») (World Health Organization 2011a). 
américains se seraient vu interdire l'adoption d'enfants de la région ${ }^{14}$.

Ces exemples, de l'ordre des représentations sociales, illustrant toutes sortes de craintes, soulignent l'assomption du lien entre " population autochtone » et alcoolisme dans la région, justifiée par des représentations populaires d'ordre médical, notamment sur la santé mentale des enfants nés de mère alcoolique.

Les nombreuses églises évangéliques implantées dans la région depuis les années 1990 sont marquées, comme le reste de ces mouvements dans le monde, par l'importance de la double conversion : la rupture évènementielle selon une définition paulinienne de la conversion avec un " avant » et un " après » identifiable, et la rupture permanente ou sans cesse renouvelée liée à la vie post-conversion. Cette conversion s'appuie sur l'identification de «fléaux » qui font l'objet de combats incessants pour le croyant ou le nouveau converti (Coleman 2004 ; Robbins 2004a ; Robbins 2004b ; Alvarsson 2003 ; Pelkmans 2009 ; Rambo 2003). Le combat contre l'alcoolisme est central dans les Églises évangéliques de la région de l'Amour : il prend la forme d'une abstinence totale d'alcool pour les dirigeants, et quasi-totale pour les membres de l'église ; il fait également l'objet de prêches de la part des pasteurs. Chez les membres de ces Eglises interrogés, Nanaïs et issus d'autres minorités locales (Iakoutes, Ul'chs, Nivkhs), le combat contre l'alcool fait partie d'une réelle motivation pour la conversion. Les discours de conversion, souvent construits de manière normative, organisés autour de l'avant et après conversion, avec identification des fléaux (Pelkmans 2009), sont marqués par le poids de l'alcool dans la vie de l'individu avant sa conversion.

Une femme nivkhe par exemple, s'est convertie après que son frère fut sauvé de l'alcoolisme par l'Eglise ; une femme iakoute s'est convertie après que son fils fut tué par des jeunes gens alcoolisés ; un homme nanaï, issu d'une famille monoparentale éclatée, s'est converti à la suite de la mort de plusieurs de ses frères en lien avec l'alcool. D'autre part, les échecs d'implantation des églises évangéliques dans les villages nanaïs, qui surpassent largement les réussites, sont attribués à l'alcoolisme, lui-même lié à la présence d'esprits chamaniques, du point de vue russe et nanaï. Ainsi, une jeune femme russe appartenant à une église évangélique disait que les Nanaïs ont jeté des sorts aux protestants dans un village de la région, conduisant au divorce d'un couple de pasteur et à 1 'alcoolisme du mari ${ }^{15}$.

\section{Conclusion}

L'utilisation et la consommation rituelles de l'alcool étaient d'abord liées à la négociation avec les esprits, aux chamanes et à la fête. Elles sont aujourd'hui également associées aux notions d'alcoolisme, de suicide et de dépression, portées par les représentations russes culturellement dominantes, réinterprétées dans des termes nanaïs d'isolation et d'esprits néfastes. L'alcoolisation festive qui porte chance fait miroir à l'alcoolisation-maladie liée à des contextes perçus comme « difficiles ». Tandis que la qualité et la quantité d'alcool ingéré peuvent être identiques, l'identification d'une consommation acceptable ou non semble reposer sur la « manière de boire ». Ainsi, une

14 Informations recueillies à Khabarovsk en avril 2015. Je n'ai pas été en mesure de vérifier les propos rapportés.

15 Informations recueillies à Khabarovsk, Komsomolsk-na-Amure, Najhin et Nikolaevsk-na-Amure entre 2011 et 2015 . 
consommation de «type russe », principalement le zapoi, est perçue comme négative et est souvent attribuée aux esprits et aux âmes errantes qui hantent les vivants.

La vision d'une " mauvaise » consommation est également relayée dans les représentations sociales des Nanaïs, véhiculées par eux-mêmes et par les autres groupes. Celle-ci fournit d'ailleurs une motivation aux groupes évangéliques de la région : signe d'un fléau à combattre, la consommation d'alcool justifie, d'un point de vue émique, aussi bien la conversion que le travail social.

La nuance consécutive à ces différentes perceptions, entre consommation acceptable et inacceptable, souligne une ambivalence générale de la culture nanaïe, entre mode de vie russe et réadaptations nanaïes. Néanmoins, l'étude de l'alcoolisme chez les Nanaïs permet de les inscrire dans un phénomène qui dépasse ce groupe dans une question beaucoup plus large, celle du suicide et de l'alcoolisme en Russie et parmi les « populations autochtones » de l'espace russophone.

\section{Références citées}

Alvarsson, Jan Ake, 2003. "A Few Notes on Conversion to Pentecostalism Especially among Ethnic Minority Groups", in Jan Ake Alvarsson \& Rita Laura Segato (eds), Religions in Transition: Mobility, Merging and Globalization in the Emergence of Religious Adhesions, pp. 33-64. Uppsala: Uppsala Universitet.

AtKInson, Thomas Witlam, 2001. Travels in the Regions of the Upper and Lower Amoor. New Delhi: Bhavana Books and Prints.

BACON, Elizabeth E., 1980. Central Asians Under Russian Rule: A Study in Cultural Change. Ithaca (NY): Cornell University Press.

Balzer, Marjorie M., 1999. The Tenacity of Ethnicity: a Siberian Saga in Global Perspective. Princeton (NJ): Princeton University Press.

Berelowitch, Wladimir, 1981. «Les débuts du droit de la famille en RSFSR : Pourquoi et comment ?», Cahiers du monde russe et soviétique, 22 (4), pp. 351-374.

BobaK, Martin, Martin McKee, Richard Rose, et Mickael Marmot, 1999. "Alcohol Consumption in a National Sample of the Russian Population”, Addiction, 94 (6), pp. 857-866.

Bodin, Jean, 1990. La coutume (4). Paris : De Boeck Supérieur.

BogojaVlenskiJ, Dimitri. D., 2008. Narody Severa rossii: demograficheskij profil' na rubezhe vekov [les peuples du Nord de la Russie: profil démographique à la fin du siècle]. Moscou : Institut Demografii.

Bulgakova, Tatiana, 2003. "Nanaï Shamans under Double Oppression. Was the Persecution by Soviet Power Stronger than the Power of Shamanistic Spirits?", Proethnologia, 15, pp. 131-158

Bulgakova, Tatiana, 2008. "Revival of Christianity and Shamanism among the Indigenous Peoples of Siberia”, in Olivier LardinoIs et Benoit Vermander (eds), Shamanism and Christianity, Religious Encounters among Indigenous People of East Asia, pp. 145-162. Taipei: Taipei Ricci Institute.

Bulgakova, Tatiana, 2013. Nanai Shamanic Culture in Indigenous Discourse. Germany, Norderstedt: SEC publications.

Coleman, Simon, 2004. The Globalization of Charismatic Christianity: Spreading the Gospel of Prosperity. Cambridge: Cambridge University Press.

GAER, Evdokia. A., 1983. « Pogrebenie rebenka u Nanajcev [enterrements des enfants chez les Nanaïs] » in L.U. Rusova (éd.), Tradicii i sovremennost'v kul'ture narodov Dal'nego Vostoka, Dal'nevostochnogo nauchnogo centra AN SSSR, pp. 44-48. Vladivostok : Dal'nevostochnogo nauchnogo centra AN SSSR

GAER, Evdokia. A, 1991. Tradicionnaja bytovaja obrjadnost' nanajcev v konce XIX načale XX $v$ [rites domestiques traditionnels nanaïs à la fin du 19e-début 20 siècles] Moscou : Mysl'.

HAAs, Paula, 2014. "Contradictory Moralities: Alcohol Consumption in Inner Mongolia", Asian Anthropology, 13 (1), pp. 20-35. 
Hinton, Devon E., et Roberto Lewis-Fernandez, 2010. “'Idioms of Distress' (Culturally Salient Indicators of Distress) and Anxiety Disorders", in Helen B. Simpson, Yuval Neria, Roberto Lewis-FernandeZ et Franklin SchneIER (eds), Anxiety Disorders: Theory, Research and Clinical Perspectives, pp. 127138. Cambridge: Cambridge University Press.

Istomin, Kirill, 2012. "Once Again on the Problem of Alcoholism and Suicide among the Indigenous Peoples of the Russian North. Can Attribution Style Be a Factor?”, Sibirica 11(3), pp.1-19.

Keys, Hunter M., Bonnie N. Kaiser, Brandon A. Kohrt, Nayla M. Khoury et Aimée-Rika T. Brewster, 2012. "Idioms of Distress, Ethnopsychology, and the Clinical Encounter in Haiti's Central Plateau", Social Science \& Medicine, 75 (3), pp. 555-564.

Kolotilin, Genadij. F., Aleksandr, A. Andrejanov, Dimitrii N. Gololobov, et Maksim. A. Goncharov, 1998. « Opyt raboty suicidologicheskoj sluzhby Xabarovskogo gorodskogo psixoterapevticheskogo centra. [Expérience de travail dans le centre de suicidologie du centre de psychothérapie de la ville de Khabarovsk]», in V.I. MinaJlov, B.S. Polozhis, et A.A. Čurkin (éds), Aktual'nye problemy psixiatrii, narkologii i nevrologii, pp. 20-24. Khabarovsk : Sbornik nauchnyx trudov.

Kolotilin, Genadij F., et Ju D. KRUDRJAVCEV, 1998. «Psihopatologicheskij variant alkogolizma u aborigenov Priamur'ja. [Variantes psychopathologiques de l'alcoolisme chez les aborigènes du Priamur'je] », in V.I. Mihajlov, B.S. Polozhis, et A.A. ČURKIn (éds), Aktual'nye problemy psixiatrii, narkologii $i$ nevrologii, pp.144-149. Khabarovsk: Sbornik nauchnyx trudov.

Kosareva, S.P., et S.M. MostovoJ, 1998. « Sravitel'naja xarakteristika upotreblenija gashisha i jefredona russkimi i aborigenami Dal'nego Vostoka. [Caractéristiques comparées de l'utilisation de haschisch et d'éphédrine chez les Russes et Aborigènes de l'Extrême-Orient] », in V.I. MiHAJLOV, B.S. PoLOZHIJ, et A.A. ČURKIN (éds), Aktual'nye problemy psixiatrii, narkologii i nevrologii, pp. 197-201. Khabarovsk: Sbornik nauchnyx trudov.

KosAREVA, S.P., et N.P. PANJUSHKINA, 1998. «Osobennosti opijnoj narkomanii u korennyx narodov Priamur'ja. [Particularités de la narcomanie d'opiacés chez les populations autochtones du Priamur'je]», in V.I. Mihajlov, B.S. Polozhis, et A.A. Čurkin (éds), Aktual'nye problemy psixiatrii, narkologii $i$ nevrologii, pp. 201-204. Khabarovsk : Sbornik nauchnyx trudov.

Kossov, Valéry, 2014. « Boire ou ne pas boire? Des représentations autour de l'alcool dans le discours politique russe », ILCEA, 19, pp. 10.

Kossov, Valéry, 2015. « La Doxa et l'alcool : le rôle des représentations historiques et culturelles dans le discours du pouvoir russe. », La Revue Russe, 44, pp. 185-196.

LaCAZE, Gaëlle, 2004. «Convivialité et consommation d'alcool chez les Mongols et les Kazakhs », Annales de la Fondation Fyssen, 19, pp. 30-47.

LÉVI-Strauss, Claude, 1967. Les structures élémentaires de la parenté. Paris : Mouton \& Co.

Lopatin, Ivan A., 1960. The Cult of the Dead among the Natives of the Amur Basin. Gravenhage: Mouton \& Co.

MASsÉ, Raymond, 1999. « La santé publique comme nouvelle moralité », in Pierre ForTIN (dir.), La réforme de la santé au Québec, pp. 155-174. Montréal : Les Éditions Fides.

MAssé, Raymond, 2007. « La 'troisième voie' en anthropologie de la santé : pour une réflexivité constructive », in Olivier LeSERVOISIER et Laurent VIDAL (dir.), L'anthropologie face à ses objets. Nouveaux contextes ethnographiques, pp. 267-281. Paris : Éditions des Archives contemporaines.

McKeE, Martin, 1999. “Alcohol in Russia”, Alcohol and Alcoholism, 34 (6), pp. 824-829.

MerLi, Laetitia, 2004a. "Chinggis Khan and the New Shamans”, Inner Asia, 6 (1), pp. 119-124.

Merli, Laetitia, 2004b. De l'ombre à la lumière, de l'individu à la nation : Renouveau du chamanisme en Mongolie postcommuniste. Thèse de doctorat. EHESS.

Mostovoj, S.M., et Boris S. PolozhiJ, 1998. « Jetnokul'turnye osobennosti rasprostranennosti alkogolizma i alkogol'nyx psixozov v Xabarovskom krae v 1996-1997 godax. [Particularités ethnoculturelles de la prévalence de l'alcoolisme et de la psychose alcoolique dans le kraj de Khabarovsk en 1996 et 1997] », in V.I. Mihajlov, B.S. PolozhiJ, et A.A. ČUrKIN (éds), Aktual»nye probelmy psihiatrii, narkologii i nevrologii, pp. 153-59. Khabarovsk : Sbornik nauchnyx trudov.

Nemtsov, Alexander. V., 2000. "Estimates of total alcohol consumption in Russia, 1980-1994", Drug and Alcohol Dependence, 58, pp. 133-142. 
Nemtsov, Alexander V., 2009. Alkogol'naja istorija rossii. Novejshij period [Histoire de l'alcool en Russie. Période moderne]. Moscou : Knizhnyj dom "Librokom"

Nemtsov, Alexander, V. 2011. A Contemporary History of Alcohol in Russia. Stockholm: Södertörns högskola.

NiCHTER, Mark, 1981. "Idioms of Distress: Alternatives in the Expression of Psychosocial Distress: A Case Study from South India", Culture, Medecine and Psychiatry, 5 (4), pp. 379-408.

OBADIA, Lionel, 2004a. « Le "boire”, une anthropologie en quête d'objet, un objet en quête d'anthropologie », Socio-anthropologie, 15 , pp. 2-14.

OBADIA, Lionel, 2004b. «"No King”, "No drink", "Power to the People" : rébellion maoïste et conduites d'alcoolisation dans le Népal contemporain », Socio-anthropologie, 15, pp. 2-9.

Pelkmans, Mathijs, 2009. "Introduction: Post-Soviet Space and the Unexpected Turns of Religious Life", in Mathijs Pelkmans (ed.), Christian Conversion after Socialism: Disruptions, Modernisms and the Technologies of Faith, pp. 1-16. New York: Berghahn

PIKA, Alexander, Eugenia W. DAvis et Igor I. KRUPNIK, 1993. "The Spatial-Temporal Dynamic of Violent Death among the Native Peoples of Northern Russia", Arctic Anthropology, 30 (2), pp. 61-76.

PIVNEVA, E.A, 2005. « Jetnograficheskie aspekty problemy alkogolizma u malochislennyx narodov severa. [Aspects ethnographiques des problèmes de l'alcoolisme chez les populations minoritaires du Nord] », in B.O. Dolgih (éd.), $V$ poiskax sebja: Narody severa i sibiri v postsovetskix transformacijax, pp. 65-84. Moscou : Rossijskaja akademija nauk.

Pozolotin, Sergej. I., éd. 2014. « Sostojanie zdravooxranenija v xabarovskom krae. Stat. Sbornik. [Etat de santé dans le kraj de Khabarovsk. Collection statistique.] ", in S. Pozolotin, (éd.), Federal'naja sluzhba gosudarstvennoj statistiki. Territorial'nyj organ federal'noj sluzhby gosudarstvennoj statistiki po khabarovskomu kraju, pp. 130. Khabarovsk : Khabarovskstat.

RAmbo, Lewis, 2003. "Anthropology and the Study of Conversion", in Andrew S. BuCKSER \& Stephen D. Glazier (eds), The Anthropology of Religious Conversion, pp. 211-222. Lanham: Rowman \& Littlefield.

Robbins, Joel, 2004a. Becoming Sinners: Christianity and Moral Torment in a Papua New Guinea society. Berkeley: University of California Press.

Robbins, Joel, 2004b. "The Globalization of Pentecostal and Charismatic Christianity", Annual Review of Anthropology, 33, pp. 117-143.

RYAN, Mickael, 1995. "Russian Report: Alcoholism and Rising Mortality in the Russian Federation”, British Medical Journal, 310, pp. 646-648.

SAmar, Ermizh, 2003. Pod sen'ju rodovogo dereva [Dans l'ombre de l'arbre clanique]. Khabarovsk : Xabarovskogo knizhnoe izdatel'stvo.

SchePer-Hughes, Nancy et Margaret M. Locke, 1987. "The Mindful Body: A Prolegomenon to Future Work in Medical Anthropology", Medical Anthropology Quarterly, New Series 1 (1), pp. 6-41.

Schrad, Mark Lawrence, 2014. Vodka Politics: Alcohol, Autocracy, and the Secret History of the Russian State. New York: Oxford University Press.

Shimkevich, Petr P., 1896. Materialy dlja izučenija šamanstva u gol'dov [Matériaux pour l'étude du chamanisme chez les Goldes]. Numéro special Zapiski priamurskogo otdela russkogo geografičeskogo obščestva. Khabarovsk.

Slezkine, Yuri, 1994. Russia and the Small Peoples of the North: Arctic Mirrors. Ithaca \& Londres: Cornell University Press.

SmoljaK, Anna V., 1968. « Pogrebal'nye i pominal'nye obrjady u narodov Nizhnego Amura i problemy preodolenija religioznyx perezhitkov. [Rites d'enterrements et commémoratifs chez les populations du Bas Amour et les problèmes de l'élimination des survivances religieuses] », in A.P. OKLADNIKO et D.D. LuBSANOV (éds), Voprosy preodolenija perezhitkov proshlogo i stanovlenija novyx obychaev $i$ tradicij, 1, pp. 157-164. Ulan-Udje : Burjatskoe knizhskoe izdatel'stvo.

Smoljak, Anna V., 1975, Etničeskie processy u narodov Nižnego Amura i Sahalina [Processus ethniques chez les populations du Bas Amour et de Sakhaline]. Moscou : Nauka. 
Smoljak, Anna V., 1980a, « Poxoronnaja obrjadnost' : Nanajci, Ul'chi, Negidal'ci, Orochi, Udjegejci \& Nivkhi [Rituels funéraires: Nanaïs, Ul'chs, Négidals, Orochs, Udéghés et Nivkhs] », in I.S. GuRVICH (éd.), Semejnaja obrjadnost' narodov Sibiri, pp. 177-199. Moscou : Nauka.

SmolJaK, Anna V, 1980b. « Svabednaja obrjanost' : Nanajci, Ul'chi \& Nivkhi [Rituels de mariages: Nanaïs, Ul'chs et Nivkhs] », in I.S. GURVICH (éd.), Semejnaja obrjadnost'narodov Sibiri, pp. 61-78. Moscou : Nauka.

SmoljaK, Anna V., 1991. Šaman: ličnost' funkcii mirovozzrenie (narody Nižnego Amura) [Chamane : personnage, fonctions, vision du monde (peuples du Bas Amour)]. Moscou : Nauka

SmoljaK, Anna V., 2005. « Ob jekonomike, kul'ture i byte korennogo naselenija Nanajskogo, Komsomol'skogo, Ul'čskogo, Sovgavan'skogo rajonov Xabarovskogo Kraja (1961g) [Sur la culture économique et quotidienne des populations autochtones des Rajons nanaïs, de Komsomolsk, ul'ch, de sovgavan dans le kraj de Khabarovsk] », in Z. P. SoKolovo et M. Pivneva (éds), Jetnologičeskaja jekspertiza, narody severa rossii 1959-1962 gody, pp. 299-323. Moscou : Institut Jetnologii i Antropologii RAN.

Solodun, Yuriy V., Yulia B. Monakhova, Thomas Kuballa, Andriyv Samokhvalov et Dirk W. LACHENMEIER, 2011. "Unrecorded Alcohol Consumption in Russia: Toxic Denaturants and Disinfectants Pose Additional Risks”, Interdisciplinary Toxicology 4 (4), pp. 198-205.

Sternberg, Lev A., 1933. Giljaki, Oroči, Gol'di, Negidal'ci, Ajny [les Gilyaks, Orochs, Négidals, Aïnous]. Khabarovsk : Dal'giz.

SternberG, Lev A, 1999. "The Social Organisation of the Gilyak”, in Bruce Grant (ed.), Anthropological Papers of the AMNH 82, pp. 280. Washington: University of Washington Press.

Sumarokov, Yuri, Tormod Brenn, Alexander Kudryavtsev et Odd Nilssen, 2014. "Suicides in the indigenous and non-indigenous populations in the Nenets Autonomous Okrug, Northwestern Russia, and associated socio-demographic characteristics", International Journal of Circumpolar Health, 73, pp. 1-8.

TAKala, Irina. R., 2002. Veselie Rusi. Istorija alkogol'noj problemy v Rossii. [Joyeuse Russie. Histoire du problème alcoolique en Russie]. Saint-Pétersbourg : Zhurnal "Neva".

TremL, Vladimir, 1997. "Soviet and Russian Statistics on Alcohol Consumption and Abuse", Social Science Research Network, pp. 1-38

« Vserossijskaja perepis' naselenija 2010 goda [Recensement de la population de toute la Russie en 2010] », 2010,<http:/www.gks.ru/free_doc/new_site/perepis2010/croc/perepis_itogi1612.htm>

Walberg, Peder, Martin McKee, Vladimir Shkolnikov, Laurent Chenet et David A. Leon, 1998. "Economic Change, Crime and Mortality Crisis in Russia: Regional Analysis", British Medical Journal, 317 (août), pp. 312-318.

White, Stephen, 1996. Russia Goes Dry: Alcohol, State and Society. Cambridge: Cambridge University Press.

World Health Organization, 2011a. « Fœtopathie alcoolique : espoirs brisés et vies gâchées », Bulletin de l'Organisation Mondiale de la Santé, 89, pp. 398-399.

WHO, 2011b. Global Status Report on Alcohol and Health. Genève : World Health Organization.

WHO, 2014. "Russian Federation. Alcohol Consumption: Levels and Patterns", < http://www.who.int/ substance_abuse/publications/global_alcohol_report/profiles/rus.pdf $>$

ZIKer, John P., 2002. Peoples of the Tundra: Northern Siberians in the Post-Communist Transition. Long Grove (IL): Waveland Press Inc.

Zolotarev, Aleksander M., 1939. Rodovoj stroj i religija ul'chej [Construction clanique et religion ul'chs]. Khabarovsk : Dal'giz. 